\title{
Article \\ Artificial Neural Networks Combined with the Principal Component Analysis for Non-Fluent Speech Recognition
}

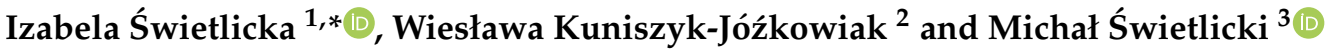 \\ 1 Department of Biophysics, University of Life Sciences, Akademicka 13, 20-950 Lublin, Poland \\ 2 Faculty of Physical Education and Health in Biała Podlaska, Józef Piłsudski University of Physical Education \\ in Warsaw, Akademicka 2, 21-500 Biała Podlaska, Poland; wieslawa.jozkowiak@awf.edu.pl \\ 3 Department of Applied Physics, Faculty of Mechanical Engineering, Lublin University of Technology, \\ Nadbystrzycka 36, 20-618 Lublin, Poland; m.swietlicki@pollub.pl \\ * Correspondence: izabela.swietlicka@up.lublin.pl
}

Citation: Świetlicka, I.;

Kuniszyk-Jóźkowiak, W.; Świetlicki, M. Artificial Neural Networks

Combined with the Principal

Component Analysis for Non-Fluent Speech Recognition. Sensors 2022, 22, 321. https://doi.org/10.3390/ s22010321

Academic Editor: Steve Ling

Received: 1 December 2021

Accepted: 27 December 2021

Published: 1 January 2022

Publisher's Note: MDPI stays neutral with regard to jurisdictional claims in published maps and institutional affiliations.

Copyright: (C) 2022 by the authors. Licensee MDPI, Basel, Switzerland. This article is an open access article distributed under the terms and conditions of the Creative Commons Attribution (CC BY) license (https:// creativecommons.org/licenses/by/ $4.0 /)$

\begin{abstract}
The presented paper introduces principal component analysis application for dimensionality reduction of variables describing speech signal and applicability of obtained results for the disturbed and fluent speech recognition process. A set of fluent speech signals and three speech disturbances-blocks before words starting with plosives, syllable repetitions, and sound-initial prolongations-was transformed using principal component analysis. The result was a model containing four principal components describing analysed utterances. Distances between standardised original variables and elements of the observation matrix in a new system of coordinates were calculated and then applied in the recognition process. As a classifying algorithm, the multilayer perceptron network was used. Achieved results were compared with outcomes from previous experiments where speech samples were parameterised with the Kohonen network application. The classifying network achieved overall accuracy at $76 \%$ (from $50 \%$ to $91 \%$, depending on the dysfluency type).
\end{abstract}

Keywords: principal component analysis; stuttering; speech recognition; artificial neural networks

\section{Introduction}

Speech, the most important means of communication, whose main aim is to send and receive messages in the linguistic form, might be disturbed or interfered with by physiological or physical factors, e.g., stuttering, becoming unintelligible for the listener. Stuttering is a break or interruption of normal speech, such as repetition, prolongation, or interjection of syllables, sounds, words, phrases, and silent pauses or blocks [1-4]. Despite a large body of research, our understanding of stuttering still lacks consensus and stuttering assessment by humans is perceived as subjective, inconsistent, time-consuming, and prone to error [5]. The main reason is an arising discrepancy among judges who do not have any unequivocally defined criteria at their disposal [6-9] or an objective automatic recognition system, which could support their judgements [10]. Pathological speech, especially stuttering, is characterised by variations in breathing, phonation, speech speed, speech rates, rhythm and pronunciation $[3,11]$, which procure it to be much more complex. Moreover, people with speech impediments generate signals whose application in pattern recognition systems is not as effective as for clear speech [12] because a lot of information is unclear, mixed and hidden.

Generally, pattern recognition systems consist of two main components: feature analysis and pattern classification. Most state-of-the-art speech recognition systems are based on hidden Markov models (HMMs) or artificial neural networks (ANNs), or HMM and ANN hybrids [12-15]. Neural networks play an important role both in speech [15-17] and speaker recognition [18-21], mainly due to the development of new neural network topologies as well as training and classification algorithms [14,22,23]. They have also been 
used for tasks such as classification [12,24,25] or feature extraction [26,27], isolated word recognition [28], small and large vocabulary and continuous speech recognition [29,30], as well as in disordered speech processing [7,8,12,13,31-36]. However, results achieved by recognition systems strongly depend on the input data. An adequately defined feature set could make the classification process more effective and efficient $[37,38]$, but, in some cases, e.g., stuttering, the amount of data is so huge that further analysis is complicated, and results are difficult to interpret $[39,40]$. Among the wide range of methods applied in the feature extraction process, e.g., MFCC, PLP and others, principal component analysis (PCA) might be found [37,41]. The PCA method creates new features, lower in dimension, projecting the original feature vectors into a new space. This reduction is achieved by a linear transformation to a new set of variables. New variables are uncorrelated and ordered according to their importance in representing the original variables. PCA proved useful and effective by numerous applications in engineering, biology, social science, and speech processing [41-45].

The authors of the following article decided to use the PCA algorithm for feature extraction both for the disrupted and fluent speech samples. The constructed model was then used in stuttered speech recognition with the artificial neural network application.

\section{Materials and Methods}

\subsection{The General Outline of the Experiment}

The study's objective was to test the applicability of principal component analysis in disrupted signal processing and the usefulness of achieved data in the process of disfluency recognition. Therefore, the following scheme of the experiment, presented in Figure 1, was proposed. In the first step, the speech signal was transformed with Fast Fourier Transform (FFT), 21 digital 1/3-octave filters with centre frequencies from 100 to 10,000 Hz, and an A-weighting filter. Finally, the signal took a form similar to that transmitted from the inner ear to the brain.
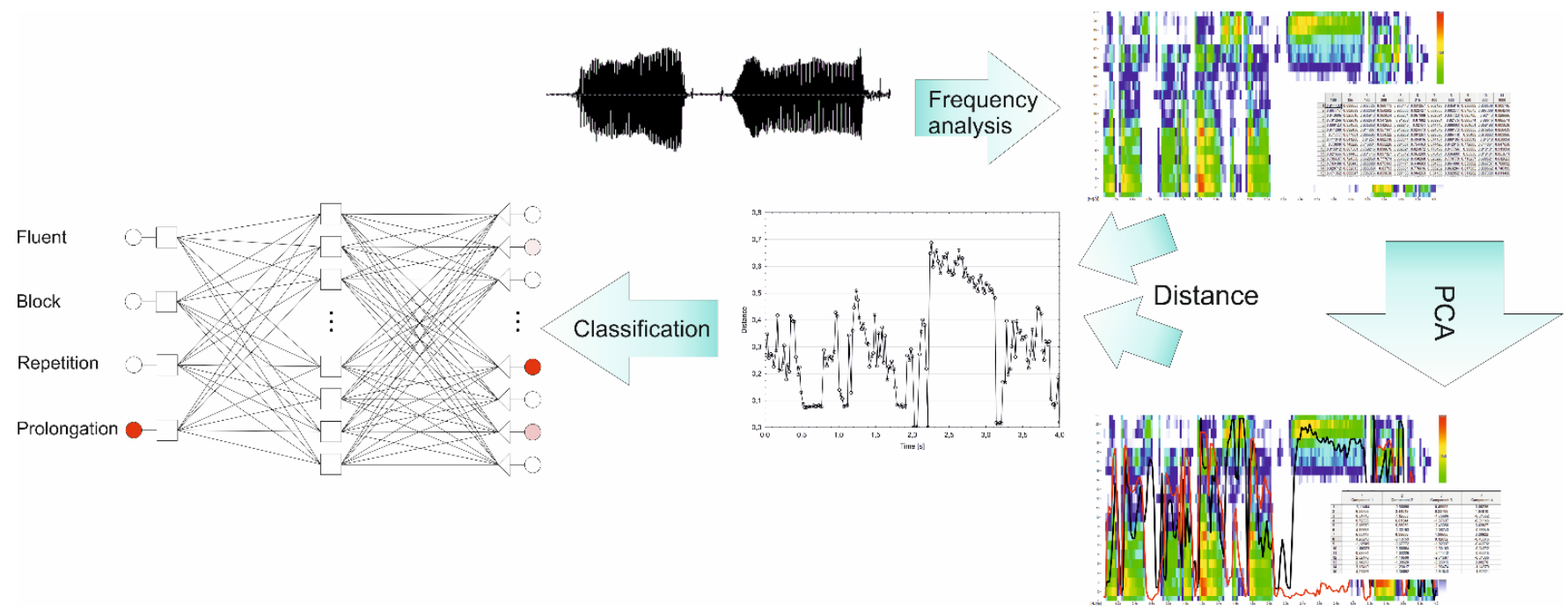

Figure 1. The experiment outline. The speech signal is transformed with FFT, $1 / 3$ octave filters, and A-filter. Next, the PCA algorithm is applied. Based on the PCA model, the distances between new (PCA) and previous system of coordinates are calculated, and then the classification process with multilayer perceptron is conducted.

The second stage of the experiment aimed to reduce the dimensionality of the vector describing each sample with the PCA algorithm application. To convert data matrix $\boldsymbol{X}=\left[x_{i j}\right]$ achieved from frequency analysis, with dimensions $[d \times m]$, where $d$ is the number of observations (171) and $m$ is the number of variables (21) into a new matrix 
$G=\left[g_{i j}\right]$ with dimension $[d \times l]$, where $l \leq m, X$ needs to be standardised according to Equation (1):

$$
z_{i j}=\frac{x_{i j}-\bar{x}}{\sigma}
$$

where $x_{i j}$-original variable element, $\bar{x}$-a mean of the original variable, and $\sigma$-original variable standard deviation. Then, correlation matrix $S$ could be defined as an $[\mathrm{m} \times \mathrm{m}]$ matrix with elements equal to correlation coefficients between variables, i.e., the product of eigenvectors $E$ and corresponding eigenvalues $\Lambda$ (Equation (2))

$$
S=E \Lambda E^{T},
$$

where: $\boldsymbol{E}=\left[\begin{array}{ccc}e_{11} & \ldots & e_{1 m} \\ \vdots & \ddots & \vdots \\ e_{m 1} & \ldots & e_{m m}\end{array}\right]$ and $\boldsymbol{\Lambda}=\left[\begin{array}{cccc}\lambda_{1} & 0 & \ldots & 0 \\ 0 & \lambda_{2} & \ldots & 0 \\ \vdots & \vdots & \ddots & \vdots \\ 0 & 0 & \ldots & \lambda_{m}\end{array}\right]$.

As seen (Equation (2)), $E$ matrix columns create eigenvectors corresponding to correct eigenvalues. If we assume that we want to have only those vectors that describe the highest part of the original data variability represented by the most significant 1 eigenvectors corresponding to the $l$ largest eigenvalues, the matrix with only $l$, instead of $\mathrm{m}$ columns, is received (Equation (3)):

$$
E_{l}=\left[e_{1}, e_{2}, \ldots, e_{l}\right],
$$

In the last step, the principal components matrix $G$ was calculated by projecting original data to new dimensions determined by eigenvectors according to Equation (4):

$$
G=Z E_{l},
$$

As a result of such an operation, matrix $[d \times l]$ was achieved, where each column contained particular principal component elements. Then, each variable from $Z$ could be described by principal components, i.e., each element of the $\boldsymbol{Z}$ matrix can be represented as a linear combination of first $l$ components. Standardised original data can be retrieved by simple data transformation (5):

$$
Y=\left(E_{l}^{T} G\right)+\bar{Z},
$$

where $Y$-original data prediction by the PCA model and $\bar{Z}$-standardized variable mean [46].

Then, distances between standardised original data matrix $\mathbf{Z}$ and corresponding elements $y_{i j}$ from the new system of coordinates were calculated according to relation (6) and used in the classification process with ANN application:

$$
d_{i}=\sqrt{\frac{\sum_{j=1}^{k}\left(z_{i j}-y_{i j}\right)^{2}}{m-l}},
$$

where: $z_{i j}$-element of the standardised original data set, $y_{i j}$ - the corresponding element of the set of observation matrix in the new system of coordinates (prediction acc. to the model), $m$-number of original variables, and $l$-number of principal components.

Calculated distances represent the residuals that allow detection of excessive distances to the model and thus map the data structure and their "shape" [46]. An observation for which the distance is equal to 0 follows the model structure, and the greater it is, the further the given observation lies from the model. Therefore, the calculated distance can be used to determine which feature the model considers as the most important and against which the entire model is built. It is a fundamental issue in constructing speech recognition models, especially concerning disfluent speech. Most of the state-of-the-art feature extraction algorithms, including Mel-frequency Cepstral Coefficients (MFCC), Linear Prediction Coefficients (LPC), Linear Prediction Cepstral Coefficients (LPCC), Discrete 
Wavelet Transform (DWT) or Perceptual Linear Prediction (PLP), are sensitive to noise and achieve excellent results for clear speech $[35,47,48]$. However, any disruption, especially prolongation, decreases their efficiency. The authors of the following article want to present a different attitude towards stuttered speech recognition, highlighting that it is not a model but the difference between the model and original data, which may indicate areas important in recognizing and classifying disturbed speech.

To test the effectiveness of the proposed solution, it was planned to make the comparison with a feature extraction method described in [31,32], where the Kohonen network was used in the process of dimensionality reduction. All the calculations and simulations were done with Statistica (TIBCO Software Inc. Palo Alto, CA, USA) application.

\subsection{Speech Samples Preparation and Processing}

One hundred and ninety-eight 4-s utterances containing three types of stuttering episodes (blocks before words starting with plosives, syllable repetitions, and sound-initial prolongations) and fluent speech were selected from the recordings of 19 people who stutter (PWS) and fluent speakers (FS), distinguished according to gender and age [31]. The recordings were made before therapy as well as during its various stages. They included two situations: reading story fragments and describing illustrations (the list of samples is placed in Supplementary Materials, Table S1). The same stories and pictures were used to record fluent samples. Patients and fluent speakers were matched according to the similarities in the signal spectrums. A female voice was recognized as equivalent to stuttering women and children, while the male voice was paired with male PWS. The detailed characterisation of the dataset is presented in Table 1.

Table 1. Characteristics of stutterers and fluent speakers regarding realised disfluency, age and gender.

\begin{tabular}{ccccccc}
\hline & \multicolumn{3}{c}{ People Who Stutter } & \multicolumn{2}{c}{ Fluent Speakers } \\
\hline Disfluency Type & Number & Gender & $\begin{array}{c}\text { Age Ranges } \\
\text { (years old) }\end{array}$ & Number & $\begin{array}{c}\text { Gender } \\
\text { Age Ranges } \\
\text { (years old) }\end{array}$ \\
\hline $\begin{array}{c}\text { blocks } \\
\text { syllable repetitions }\end{array}$ & 11 & $9 \mathrm{M}$ and 2F & $10-23$ & 8 & $4 \mathrm{M}$ and $4 \mathrm{~F}$ & $22-50$ \\
prolongations & 7 & $4 \mathrm{M}$ and 2F & $11-23$ & 6 & $4 \mathrm{M}$ and 2F & $22-53$ \\
\hline Total & $\mathbf{1 9}$ & $\mathbf{1 6 M}$ and 3F & $\mathbf{1 0 - 2 5}$ & $\mathbf{1 4}$ & $\mathbf{9 M}$ and 5F & $\mathbf{2 2 - 5 3}$ \\
\hline
\end{tabular}

$\mathrm{M}-$ male, F-female. Cited from [31].

Disfluencies were assessed in the two-stage process-firstly by four students with two years of experience at the non-fluent speech processing and next by two experts in the area of stuttering. The evaluation process aimed to select a representative sample of the particular non-fluency as far as possible without other disfluency types. The research material was recorded in an acoustic booth. The signal was transformed from an analogue to a digital form with a sampling frequency of 22,050 Hz and a sampling precision of 16 bits. Recorded samples were then analysed by the FFT 512 with a time resolution of $23 \mathrm{~ms}$. An A-weighting filter and 21 digital 1/3-octave filters of centre frequencies between 100 and $10,000 \mathrm{~Hz}$ were used.

As the result of the analysis, a $171 \times 21$ matrix for each sample was achieved. The number of vectors (171) was determined according to the time resolution, while the number of their elements (21) was due to the number of filters.

\subsection{Principal Components Analysis}

The data matrix from the previous analysis was scaled to the unitary standard deviation, which means that the PCA was done on the correlation matrix. All variables were treated on an equal footing. Principal components were determined with the use of v-fold cross-validation. Individual features of the utterances (timbre, fundamental frequency, levels of formants) influenced the number of principal components, which varied among 
the samples from 4 to 7 . To select the optimum number of variables, both the scree plots and the Kaiser-Guttman criterion were used, showing that those principal components are significant for which eigenvalues are greater than unity and explain at least $75 \%$ of the total variability. In this manner, the number of principal components was reduced to $l=4$ for all analysed utterances. Finally, 198 samples were transformed to a new system of coordinates, whereas each sample was represented by $[171 \times 4]$ matrix. The distances between transformed data and eigenspace were calculated based on the assumption that PCA also helps distinguish classes [40]. Received distance vectors, in dimensions [171 $\times 1$ ] for each sample, were gathered in one $[198 \times 171]$ matrix and used in the classification process.

\subsection{Kohonen Network Application}

The same 198 vectors built with frequency analysis results were transformed by the Kohonen network. Since SOM (Self-organising Map, Kohonen network) can detect the most important features, it was assumed that its application provides the possibility of converting the sound signal into a character string that describes the examined utterance. Therefore, the network aim was to transform a $171 \times 21$ matrix into a $171 \times 1$ matrix, where 171 represents time points, 21 is the number of $1 / 3$ octave filters, and 1 is the column of winning neurons at particular time points. The self-organising Kohonen network, built with $5 \times 5$ output neurons, trained through 100 epochs with a stable learning rate (0.1) and neighbourhood decreasing from 3 to 0 , was used. As the result of the conducted analysis, the output matrix $198 \times 171$ consisting of neurons winning in a particular time frame was obtained and used in the classification process collaterally with the matrix received from the PCA analysis. The mentioned method was widely described in [31].

\subsection{Recognition Process and Results Assessment}

Multilayer perceptron (MLP) was trained on the data representing 55 blocks, 46 syllable repetitions, 59 sound-initial prolongations and 38 fluent utterances, divided randomly into three groups-training, validation and test, as shown in Table 2.

Table 2. Data distribution according to the disfluency type and affiliation to the training set.

\begin{tabular}{ccccc}
\hline & \multicolumn{3}{c}{ The Number of Samples } & \multirow{2}{*}{ Total } \\
\hline Sample Type & Training Set & Validation Set & Test Set & \\
\hline blocks & 37 & 9 & 9 & 55 \\
syllable repetitions & 36 & 5 & 5 & 46 \\
prolongations & 42 & 5 & 12 & 59 \\
fluent & 25 & 10 & 3 & 38 \\
\hline Total & 140 & 29 & 29 & $\mathbf{1 9 8}$ \\
\hline
\end{tabular}

The network was supposed to distinguish among three disfluencies and fluent speech, so it has 171 inputs and four outputs. The number of hidden layers and neurons in each was determined based on the growth method and amounted to one hidden layer with eight neurons. The classifying network was trained with Broyden-Fletcher-Goldfarb-Shanno (BFGS) algorithm [49] for 100 epochs, with a 0.1 learning rate and constant momentum equal to 0.3. As an error function, the cross-entropy (CE) was applied (Equation (7)) because $C E$ implementation allows interpreting output values as the probabilities of the object group membership:

$$
E_{C E}=-\sum_{i=1}^{N} y_{i}\left(\frac{d_{i}}{y_{i}}\right) \text {, }
$$

where: $y_{i}$-real output value, $d_{i}$-expected output value, and $N$-the number of teaching pairs input-output. Classification results were then compared with those achieved by the same classifier taught with the same parameters and working on the data received from the 
SOM method. Finally, selected classifiers were assessed with classification accuracy (acc) (Equation (8)) and overall error rate $(\varepsilon)$ determined based on a testing set (Equation (9)):

$$
\begin{gathered}
a c c=\frac{N_{c}}{N_{t}}, \\
\varepsilon=1-a c c,
\end{gathered}
$$

where: $N_{c}$ - correctly classified cases from the test set and $N_{t}$ - the number of all test cases.

\section{Results and Discussion}

The main premise of the dimensionality reduction process is that it should be conducted to protect from losing important information, which is of the utmost importance, especially when it comes to disfluent speech recognition, where each detail could bring information needed for correct recognition or classification. Two data dimensionality reduction methods are compared: the first uses the proposed PCA transformation, while the second is based on the Kohonen network application (SOM).

\subsection{Frequency Ranges Contribution to the PCA Model}

The number of variables used to create the PCA model amounted to 21 . Due to the fact that all variables were scaled to the unitary variance, each of them had an equal chance to be represented in the model. During analysis, variables were rated according to their contribution to the model. Figure 2 presents the average contribution $(S)$ of each variable in non-fluent and fluent groups calculated according to the Equation (10) and averaged in groups:

$$
S=1-\frac{S V_{j}}{S V_{j 0}}
$$

where: $S V_{j}$ — jth variable remainder variability and $S V j_{0}-j$ th variable variability.
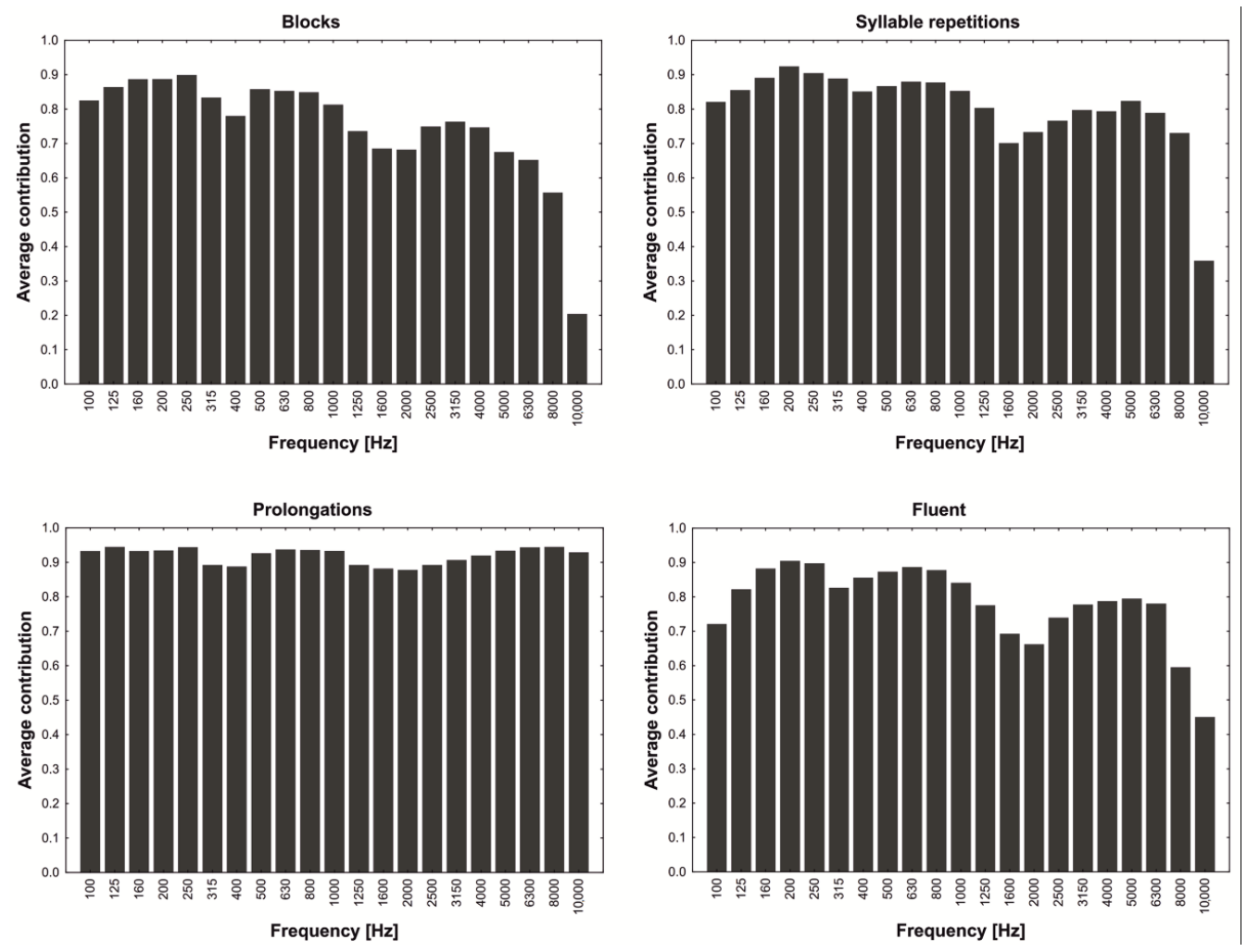

Figure 2. The average contribution of variables to the PCA model according to the analysed utterance type. 
The achieved contribution represents variables' ability to model the data-a contribution close to one means that a variable was fully used in the model. As it can be seen (Figure 2), the general course of the envelope is quite similar for blocks, syllable repetitions and fluent speech. For prolongations, the chart is of a slightly different shape. Almost all frequencies prove to have the same significance (about 0.9 ). The main difference can be noticed in the range of frequencies from 3150 to $10,000 \mathrm{~Hz}$ - those frequencies seem to be much more important for prolongations than for the rest of the analysed samples. The reason for that may be that, in the Polish language, the most often prolonged sounds are sibilants and nasals, which are characterised by a concentration of energy in the range of higher frequencies [50]. Therefore, from the shapes of the plots, it can be concluded that individual components represent some parts of the frequency structure of analysed utterances.

\subsection{The Attempt at an Interpretation of the Role of Particular Principal Components in the Description of the Speech Signal}

According to PCA theory, each component explains some part of data set variability. The first of them usually describes the greatest part of it. At the same time, each further component is chosen to ensure that it will not be correlated with previous ones and will explain most of the residual variability. As a result, each additional component explains the decreasingly lower part of variability, and, consequently, consecutive eigenvalues $e_{i j}$ are becoming lower. The signs and values of eigenvalues $e_{i j}$ explain how variable $i$ affects component $j$. However, it is more precise and easier to interpret so-called factor loadings $a_{i j}$ (Equation (11)), which also reflect the influence of particular variables $Z$ on a given principal component $G$. The higher the $\left|a_{i j}\right|$ value, the more significant the variable influence on building the principal component. If the analysis is conducted on the correlation matrix, it is possible to interpret the factor loadings as correlation coefficients between original data and particular principal components [51]. Such an approach makes it possible to interpret the 'area', which each component explains:

$$
a_{i j}=\sqrt{\lambda_{i}} e_{i j}
$$

where: $\lambda_{i}$-eigenvalue and $e_{i j}$-eigenvector corresponding to the $i$-th eigenvalue.

Trying to explain the role of each principal component, for all examined groups, the average factor loadings were calculated and illustrated in the figures, where the vertical axis gives central frequencies and the horizontal axis plots the average factor loading values (Figures 3-7). For fluent and non-fluent utterances, the average factor loadings for $\mathrm{G}_{1}$ were the highest in the frequency range from 100 to about $3000 \mathrm{~Hz}$, while $\mathrm{G}_{2}$ was mainly constructed based on frequencies from 4000 to 10,000 Hz (Figures 3-5).

Based on the obtained results, it can be concluded that the main variability of the original variables explained by the first two components divides the area of the features into lower and higher frequencies. The first component, explaining the greatest part of variability, represents low frequencies, dominant in the Polish language. In contrast, the second explains the remaining part of the variability, comprising less common, higher frequencies.

In turn, the greatest contribution to constructing the third and fourth components has particular values of frequencies, not their ranges (Figures 6 and 7). This creates specific problems with interpretation, but it can be suspected that the frequencies differ within the particular utterance groups. It is, therefore, possible that those two additional components bring more details into the general image of utterance transferring properties characterising the particular kinds of disfluency or fluent speech. 

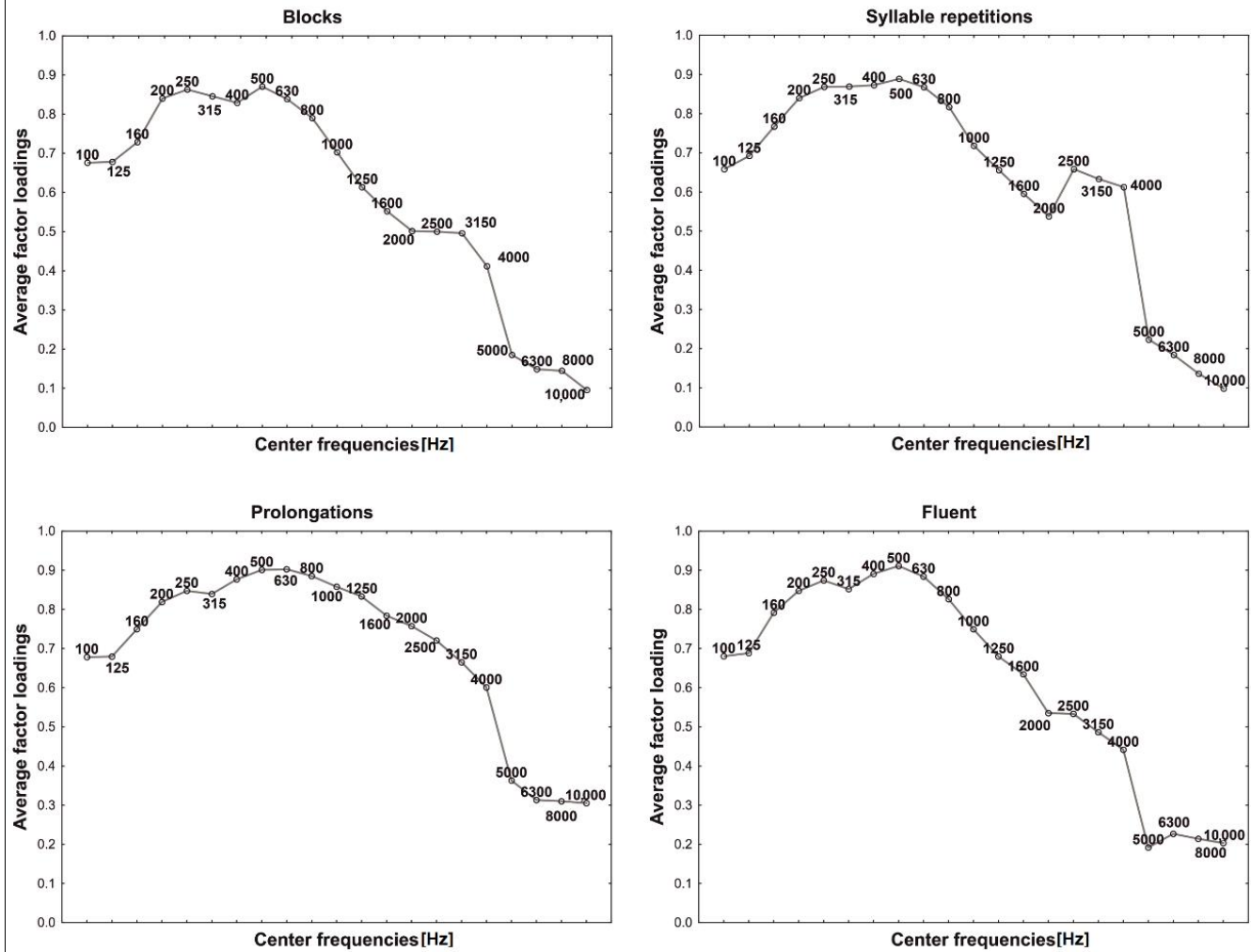

Figure 3. First component $\left(\mathrm{G}_{1}\right)$ factor loadings.
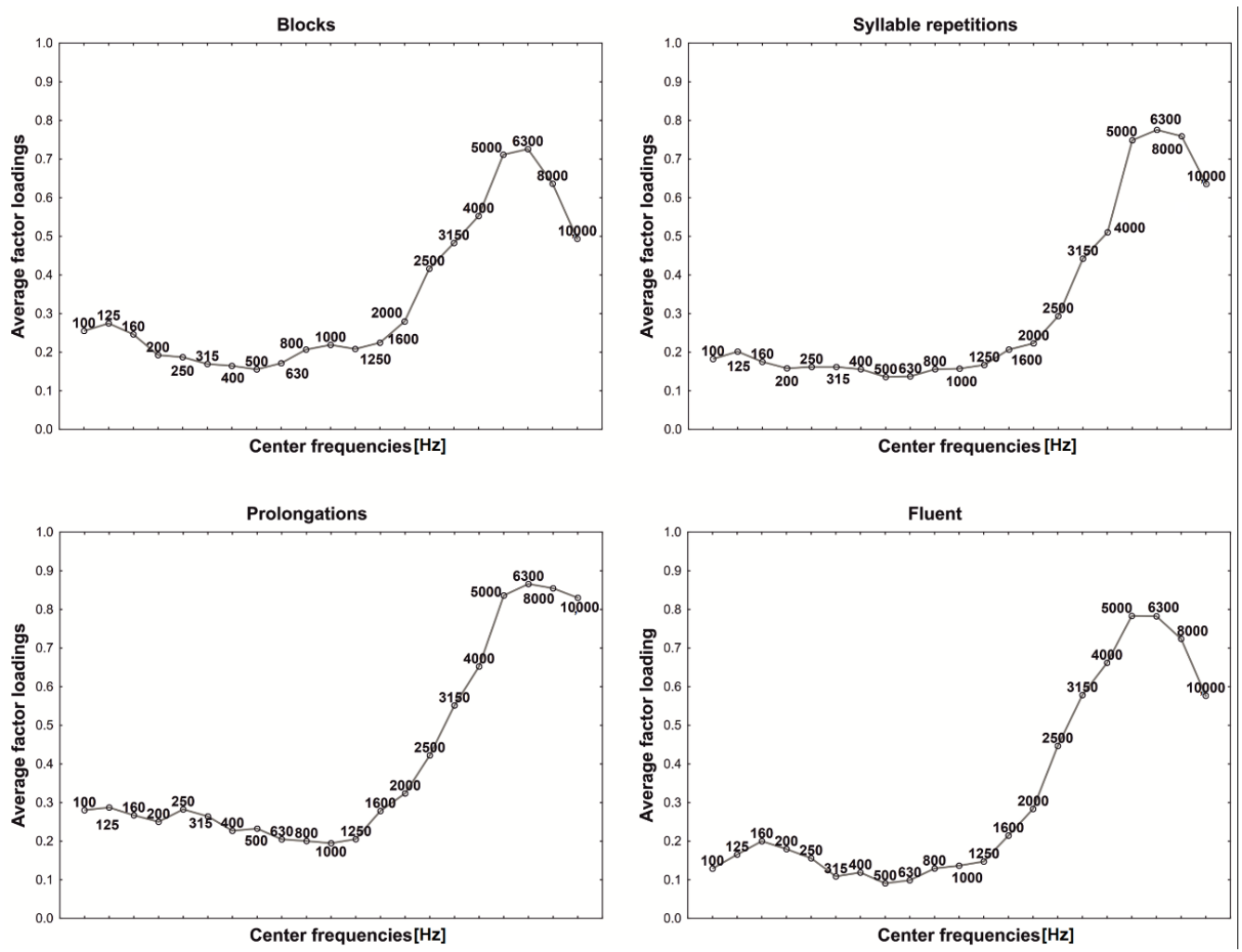

Figure 4. Second component $\left(\mathrm{G}_{2}\right)$ factor loadings. 


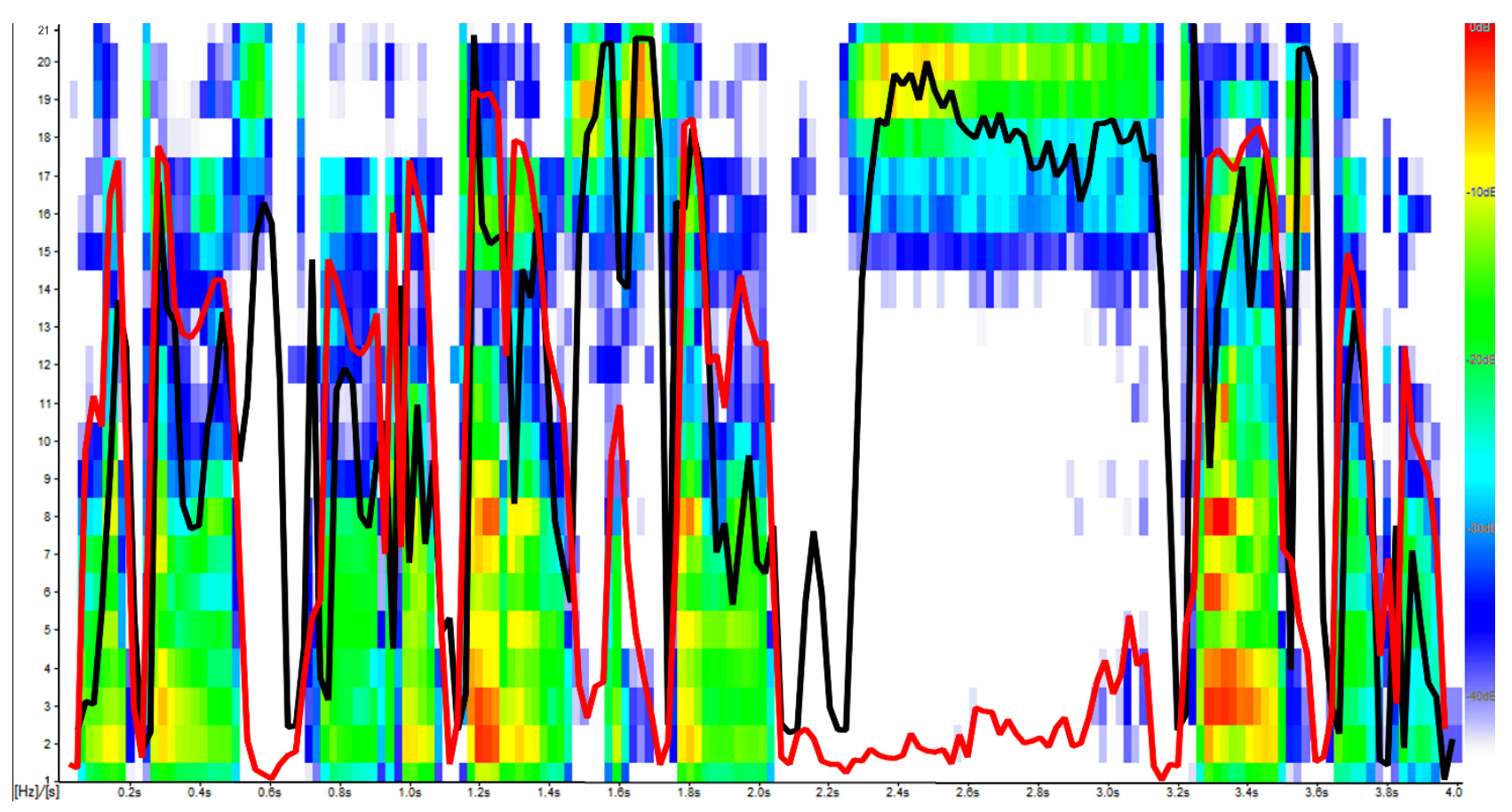

Figure 5. Prolongation spectrogram with $\mathrm{G}_{1}$ (red) and $\mathrm{G}_{2}$ (black) components. As can be observed, the shape of both G1 and G2 reflects the general time-frequency structure of the analysed utterance, but it is G2 which reflects higher frequencies while G1 concentrates on the lower ones instead.
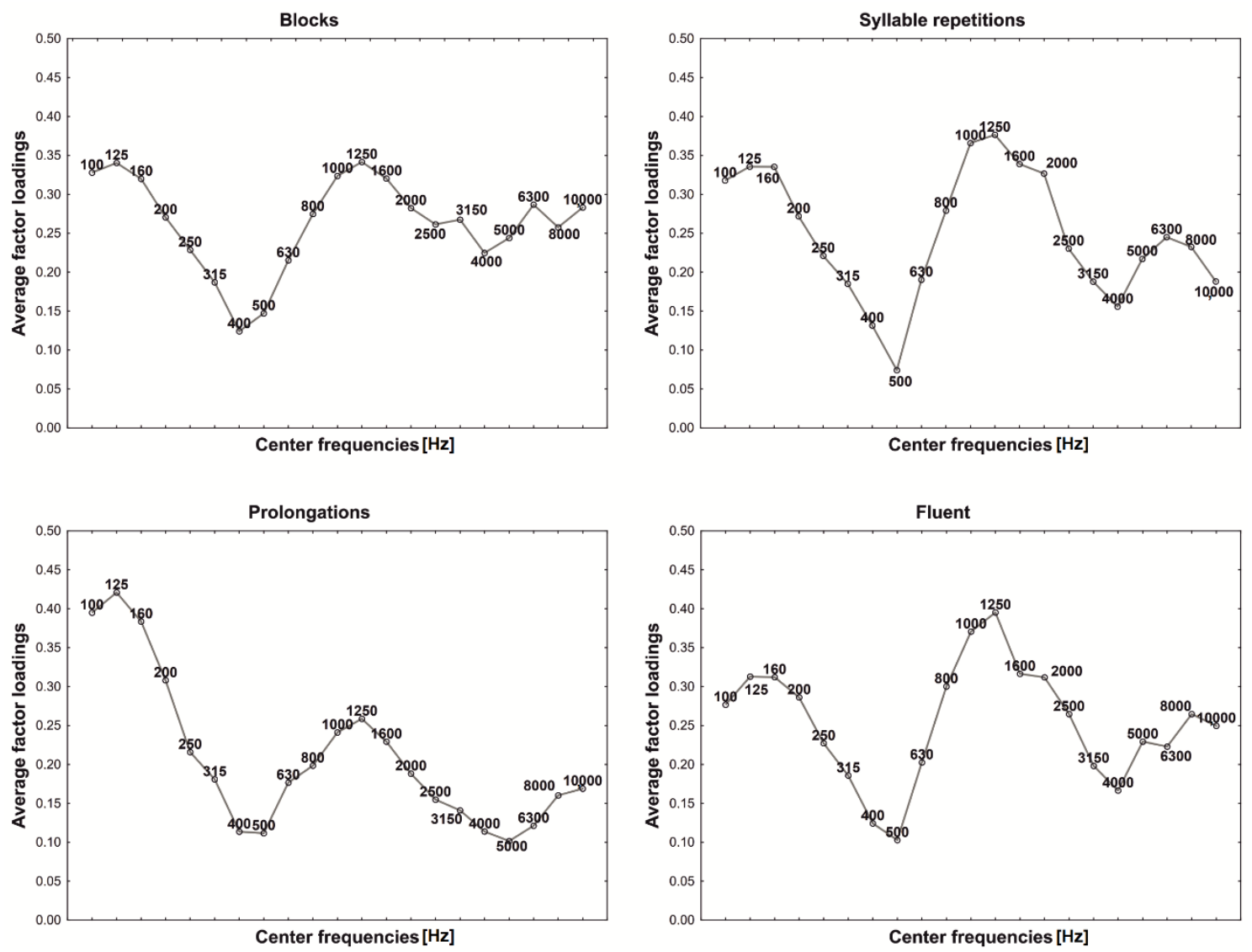

Figure 6. Third component $\left(\mathrm{G}_{3}\right)$ factor loadings. 

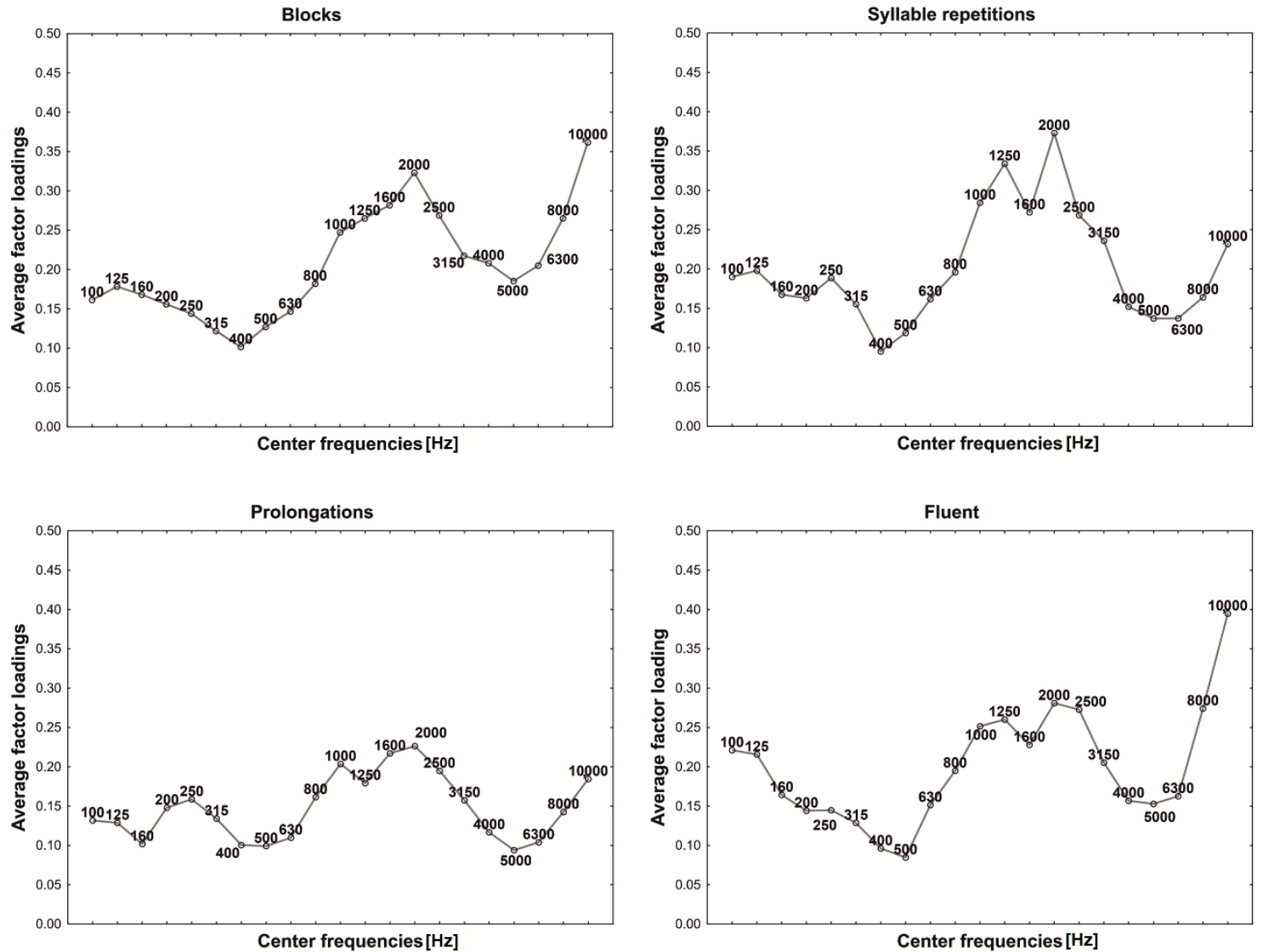

Figure 7. Fourth component $\left(\mathrm{G}_{4}\right)$ factor loadings.

In order to verify whether and which of the variables building the principal components affect the differentiation of the particular kinds of utterance, the ANOVA was applied and the Tukey RIR test for unequal groups. The results revealed that the variables included in the first component permit primarily to differentiate between fluent and nonfluent utterances $(p<0.05)$. In the case of the second component, as expected, the notable contribution of higher frequencies mainly causes prolongations to be identified $(p<0.05)$, whereas variables with the most significant contribution in building the third and fourth components permit the differentiation of utterances within the disfluencies themselves, i.e., they permit the differentiation of, e.g., a block from repetition or a prolongation from a block. However, they allow to the greatest extent to differentiate blocks $(p=0.001)$ and to the least repetitions of syllables $(p=0.041)$.

\subsection{Distance Calculation}

For obtained distance values $\mathrm{d}_{\mathrm{i}}$, plots of the distance-time relations were prepared (Figure 8a). The results showed that the smallest distance from the model was characteristic of silence fragments. In contrast, the greatest distances were noted at the moments of realisation of fragments with higher frequencies. The above observations suggest that the considered model is built based on moments of silence, i.e., absence of signal. At the same time, every other fragment of utterance already constitutes a divergence from the base model. As a result, the distances from the model become proportionally larger with relation to the frequency band characterising a given utterance fragment. Nevertheless, as observed in Figure 8a, both the syllabic structure and moments of silence were preserved. Considering previous conclusions, it might be put forward that PCA analysis reproduces the time-frequency domain of speech in significantly fewer dimensions. 
a)
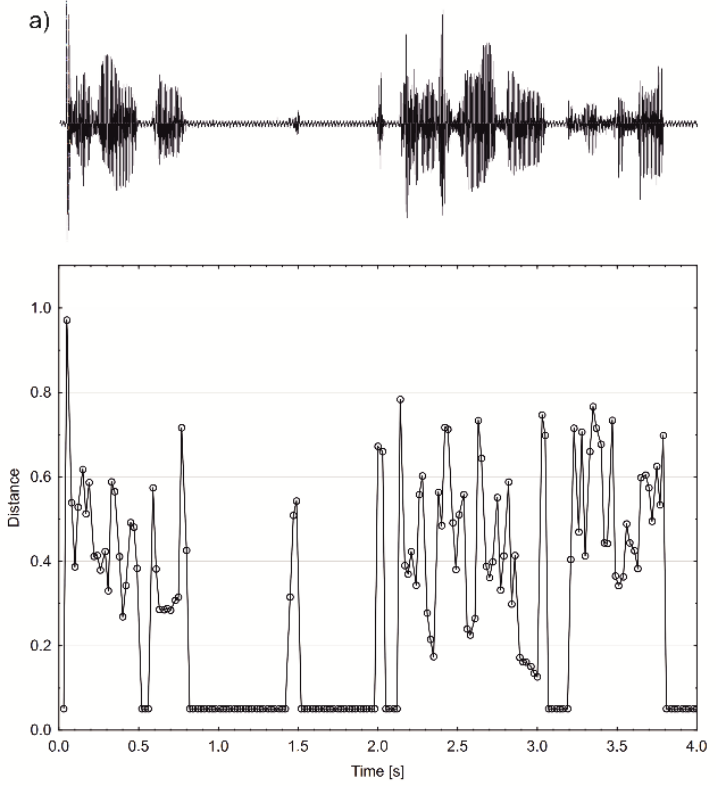

b)
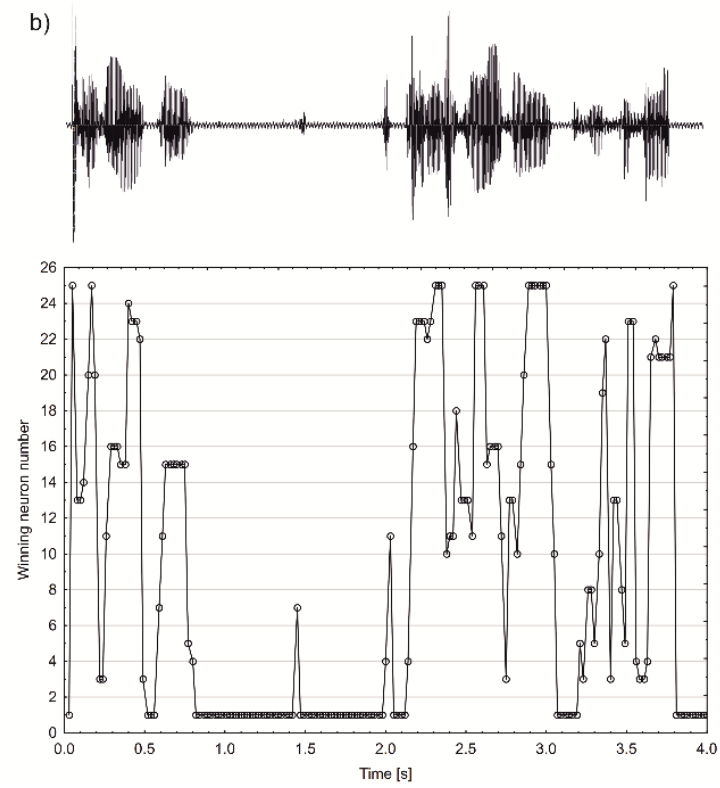

Figure 8. The representation of sound repetition with the PCA (a) and Kohonen (b) algorithm application.

The sample structures transformed with the PCA (Figure 8a) and Kohonen network (Figure 8b) show high similarity. However, slight differences could be noticed: the intensity of repetitions and detailed structure of fluent parts are relatively higher for the PCA method. In contrast, higher and lower frequencies seem to be separated to a greater degree for the Kohonen method.

\subsection{Classification Results}

The task of the MLP classifier was to isolate four groups from the presented data set: blocks, syllable repetitions, prolongations and fluent utterances. The results achieved by the classifier during the training process with the feature set received from the PCA method are presented in Table 3. For comparison, the classification results with the feature set created with the SOM method are also demonstrated.

Table 3. Recognition rates (for training, validation and test set) for an MLP classifier trained with features received with PCA and SOM application.

\begin{tabular}{cccc}
\hline \multirow{2}{*}{ Feature Extraction Method } & \multicolumn{3}{c}{ Recognition Rate } \\
\cline { 2 - 4 } & Training & Validation & Test \\
\hline PCA & 92.14 & 72.41 & 75.86 \\
SOM & 59.29 & 55.17 & 51.72 \\
\hline
\end{tabular}

As it can be seen, the same classifier trained with the use of two data sets produced results differing to a significant degree. Notably, better results were obtained for the input data resulting from PCA analysis-training, test and validation rates are about 30\% higher than the SOM extraction method's classification results. In similar research [42], where for signal dimensionality reduction, PCA was used, classifying a feedforward neural network achieved very high accuracy (close to $100 \%$ ) with 5 to 8 features, while for 3, only $67.1 \%$ was gained. However, comparing achieved results to the models where authors for stuttered speech recognition used Mel-frequency cepstral coefficients (MFCC) in the process of feature extraction [39], or MFCC, PLP and FBE features [40], the accuracy ranged between $63.5 \%$ for syllable repetitions (for eight MFCC coefficients) to $98.6 \%$ in case of interjections (for 39 MFCC coefficients). Others [52] showed that the PCA algorithm reduced the feature dimensions from 26 to 12 , resulting in the same speech recognition accuracy as the 
conventional MFCC method without PCA, and increased speech recognition accuracy for ten feature vectors.

Taking a closer look at the detailed results and comparing the values in Tables 4 and 5, it can be concluded that the classifier working on PCA data is more efficient and commits fewer mistakes than in the case of SOM features. Furthermore, a significantly higher degree of recognition was achieved in the group of blocks and prolongations (the higher of 14 and $18 \%$, respectively) and of fluent utterances, where the result was improved by $71 \%$. Only in the case of syllable repetitions did the classifier using the effects of SOM analysis prove to be more effective (difference in the range of $25 \%$ ). The presented results confirm the conclusions from Tukey's RIR test, according to which PCA analysis could be useful mainly for the differentiation between fluent utterances and prolongations, and to a lesser degree in the case of blocks.

Table 4. Classification statistics for MLP classifier calculated for the test set for PCA and SOM feature extraction methods.

\begin{tabular}{ccc}
\hline & $a c c$ & $\varepsilon$ \\
\hline PCA & 0.76 & 0.24 \\
SOM & 0.52 & 0.48 \\
\hline
\end{tabular}

Table 5. Accuracy for examined groups of disfluencies and fluent speech calculated for the test set for PCA and SOM feature extraction methods.

\begin{tabular}{ccccc}
\hline & \multicolumn{4}{c}{ Accuracy [\%] } \\
\cline { 2 - 5 } & Blocks & Syllable Repetitions & Prolongations & Fluent \\
\hline PCA & 71.43 & 50.00 & 90.91 & 71.43 \\
SOM & 57.14 & 75.00 & 72.73 & 0.00 \\
\hline
\end{tabular}

In [31], where the Kohonen network was used for feature extraction and three MLP networks were applied to classify utterances into two non-fluent and fluent groups, the accuracy achieved 84-100\% depending on the disfluency type, which is a much better result. However, it should be taken into account that the designs of the experiments differ-in the presented research, four groups were distinguished by one neural network, while, in [31], each of the three networks distinguished only between fluent and disfluent utterances. If the feature extraction method based on the Kohonen network is considered, the tested network achieved much worse results than for the vector based on PCA (Tables 4 and 5).

As it might be seen, the PCA algorithm enhances the previously proposed system for stuttered speech recognition and proved to be more effective. Recently, many reports have indicated the effectiveness of the algorithm's application for feature extraction. As shown in [53], where an adaptive moment-based backpropagation algorithm of ANN (BPVAM) has been compared with PCA combined with BPVAM in the detection of Parkinson disease based on speech data, PCA application made the system to be relatively more effective. A method that hybridizes the principal component analysis (PCA) and t-statistics for feature extraction from EEG signal was proposed in [54]. Extracted features were then used by four classifiers: support vector machine (SVM), artificial neural network (ANN), linear discriminant analysis (LDA), and k-nearest neighbour (kNN), among which ANN and SVM showed the highest classification accuracy.

The plot of the relation between classification certainty, understood as the output value for the classifying network and denoting the probability of the object belonging to a given class, and the type of utterance for the testing set (Figure 9) shows that prolongations are classified with the highest certainty, above 0.7 , followed by blocks and fluent utterances. However, the classifier does not ensure certainty concerning particular cases from the syllable repetitions group. 


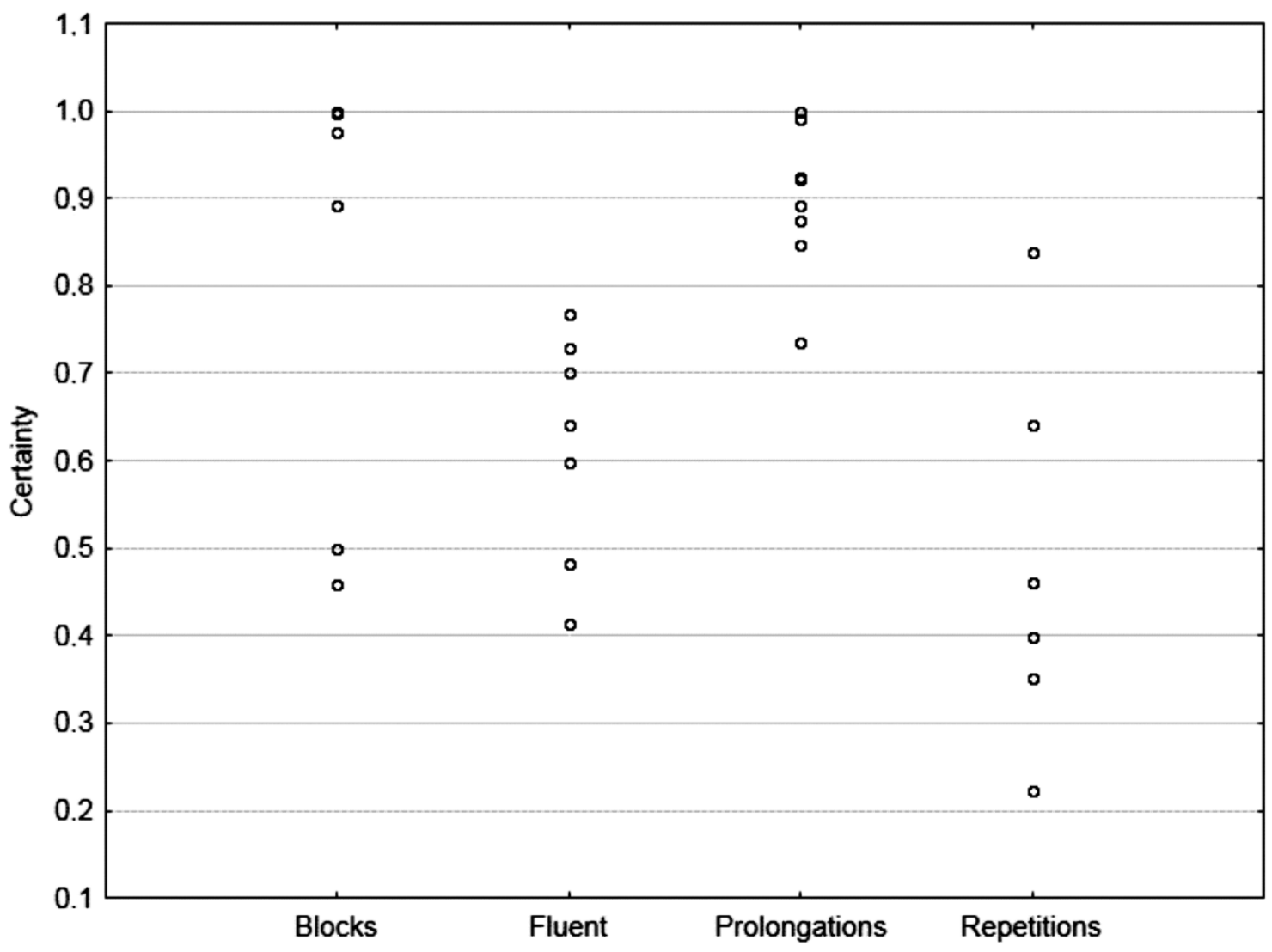

Figure 9. The certainty of classification concerning the fluency type.

While our findings provide evidence of PCA usefulness in the feature extraction process concerning stuttered speech, the presented work has some limitations which require comments. First of all, as mentioned above, not all disfluencies are pictured with the same precision, which translates into significantly worse classification results. Achieved results are probably caused by a too-small number of teaching cases within the range of this type of disfluency or incorrectness or error in describing a given kind of utterance using PCA analysis. Additionally, combining the PCA with some state-of-the-art classification algorithms, such as deep learning or Long Short-Term Memory (LSTM) and Convolutional Neural Network (CNN), which proved to be an effective tool in acoustic signal classification $[55,56]$, might enhance the results of the proposed feature extraction method. For example, in [57], it was shown that the classifier, built with a deep residual network and bidirectional long short-term memory layers, recognised different types of stutters with almost $90 \%$ accuracy. In turn, in [58], the authors presented an algorithm based on deep learning and neural networks, which proved to be helpful to diagnose stuttered speech and during therapy sessions, which seems to indicate the high potential of the methods mentioned above.

\section{Conclusions}

The presented work introduces the method where principal component analysis and artificial neural networks were applied to support the stuttered speech recognition process. The proposed solution covers the principal component analysis application as a dimensionality reduction tool, creating a new, simplified picture of disfluent speech in the frequency domain and using ANNs as classifiers. As a result, the representation of speech samples received by frequency analysis was reduced from 21 to 4 dimensions with PCA application without a significant loss of information. Furthermore, a new sample representation by four principal components preserved the utterance time structure and 
reproduced silent moments. Therefore, it could be concluded that speech signal analysis with PCA application allows for receiving speech features that could constitute the basis for its recognition. The proposed method was additionally compared with the previously developed algorithm using the Kohonen network for feature extraction. Although the approach discussed showed a significant increase in accuracy, it revealed not to be a universal method for all tested stuttering types, as detecting syllable repetitions was not impressive.

The research conducted on the principal component analysis application in a nonfluent speech analysis showed that the PCA algorithm might be treated as a valuable tool in speech processing, even a disfluent one. However, more attention and tests in the area of particular speech disorder types are needed.

Supplementary Materials: The following are available online at https://www.mdpi.com/article/ 10.3390/s22010321/s1, Table S1. Disfluent samples characteristics.

Author Contributions: Conceptualization, I.Ś. and W.K.-J.; Data curation, I.Ś.; Formal analysis, I.Ś., W.K.-J. and M.Ś.; Funding acquisition, I.Ś.; Investigation, I.Ś.; Methodology, I.Ś. and M.Ś.; Project administration, I.Ś.; Resources, W.K.-J.; Software, W.K.-J.; Supervision, W.K.-J.; Validation, W.K.-J. and M.Ś.; Visualization, I.Ś.; Writing—original draft, I.Ś.; Writing—review and editing, W.K.-J. All authors have read and agreed to the published version of the manuscript.

Funding: This research received no external funding.

Institutional Review Board Statement: Ethical review and approval were waived for this study because that database of recordings was created 30 years ago, and therefore it would be impossible to find participants to obtain their consent. Additionally, the recordings are entirely anonymous.

Informed Consent Statement: Not applicable.

Data Availability Statement: Data reported in this manuscript will be available upon request.

Conflicts of Interest: The authors declare no conflict of interest.

\section{References}

1. Howell, P.; Sackin, S. Automatic recognition of repetitions and prolongations in stuttered speech. In Proceedings of the First World Congress on Fluency Disorders, Munich, Germany, 8-11 August 1995; University Press Nijmegen: Nijmegen, Holandia, 1995.

2. Andrews, G.; Garside, R.; Harris, M. The syndrome of stuttering. In Clinics in Developmental Medicine; William Heineman Medical Books Ltd.: London, UK, 1964; Volume 17, pp. 1-191.

3. Bloodstein, O. A Handbook on Stuttering; Singular Publishing Group Inc.: San Diego, CA, USA, 1995.

4. Van-Riper, C. The Nature of Stuttering; Prentice Hall: Englewood Cliffs, NJ, USA, 1982.

5. Brundage, S.B.; Bothe, A.K.; Lengeling, A.N.; Evans, J.J. Comparing judgments of stuttering made by students, clinicians, and highly experienced judges. J. Fluency Disord. 2006, 31, 271-283. [CrossRef]

6. Howell, P.; Au-Yeung, J.; Pilgrim, L. Utterance rate and linguistic properties as determinants of lexical dysfluencies in children who stutter. J. Acoust. Soc. Am. 1999, 105, 481-490. [CrossRef]

7. Howell, P.; Sackin, S.; Glenn, K. Development of a Two-Stage Procedure for the Automatic Recognition of Dysfluencies in the Speech of Children Who Stutter: I. Psychometric Procedures Appropriate for Selection of Training Material for Lexical Dysfluency Classifiers. J. Speech Lang. Hear. Res. 1997, 40, 1073-1084. [CrossRef]

8. Howell, P.; Sackin, S.; Glenn, K. Development of a two-stage procedure for the automatic recognition of dysfluencies in the speech of children who stutter: II. ANN recognition of repetitions and prolongations with supplied word segment markers. J. Speech Lang. Hear. Res. 1997, 40, 1085-1096. [CrossRef]

9. Bothe, A.K. Identification of Children's Stuttered and Nonstuttered Speech by Highly Experienced Judges: Binary Judgments and Comparisons with Disfluency-Types Definitions. J. Speech, Lang. Hear. Res. 2008, 51, 867-878. [CrossRef]

10. Heeman, P.A.; Lunsford, R.; McMillin, A.; Yaruss, J.S. Using clinician annotations to improve automatic speech recognition of stuttered speech. In Proceedings of the INTERSPEECH 2016, San Francisco, CA, USA, 8-12 September 2016.

11. Huici, H.-D.; Kairuz, H.A.; Martens, H.; Van Nuffelen, G.; De Bodt, M. Speech rate estimation in disordered speech based on spectral landmark detection. Biomed. Signal Process. Control 2016, 27, 1-6. [CrossRef]

12. Manjula, G.; Shivakumar, M.; Geetha, Y.V. Adaptive optimization based neural network for classification of stuttered speech. In Proceedings of the 3rd International Conference on Cryptography, Security and Privacy, Kuala Lumpur Malaysia, 19-21 January 2019; Association for Computing Machinery: Kuala Lumpur, Malaysia, 2019; pp. 93-98. 
13. Narasimhan, S.; Rao, R.R. Neural Network based speech assistance tool to enhance the fluency of adults who stutter. In Proceedings of the 2019 IEEE International Conference on Distributed Computing, VLSI, Electrical Circuits and Robotics (DISCOVER), Manipal, India, 11-12 August 2019.

14. Wali, A.; Alamgir, Z.; Karim, S.; Fawaz, A.; Barkat Ali, M.; Adan, M.; Mujtaba, M. Generative adversarial networks for speech processing: A review. Comput. Speech Lang. 2021, 72, 101308. [CrossRef]

15. He, L.; Niu, M.; Tiwari, P.; Marttinen, P.; Su, R.; Jiang, J.; Guo, C.; Wang, H.; Ding, S.; Wang, Z.; et al. Deep learning for depression recognition with audiovisual cues: A review. Inf. Fusion 2021, 80, 56-86. [CrossRef]

16. Ting, H.-N.; Yong, B.-F.; MirHassani, S.M. Self-Adjustable Neural Network for speech recognition. Eng. Appl. Artif. Intell. 2013, 26, 2022-2027. [CrossRef]

17. Lei, X.; Lin, H.; Heigold, G. Deep neural networks with auxiliary Gaussian mixture models for real-time speech recognition. In Proceedings of the ICASSP 2013-2013 IEEE International Conference on Acoustics, Speech and Signal Processing, Vancouver, BC, Canada, 26-31 May 2013.

18. Zhao, X.; Wang, Y.; Wang, D. Robust Speaker Identification in Noisy and Reverberant Conditions. IEEE/ACM Trans. Audio Speech Lang. Process. 2014, 22, 836-845. [CrossRef]

19. Sarma, M.; Sarma, K.K. Speaker identification model for Assamese language using a neural framework. In Proceedings of the International Joint Conference on Neural Networks, Dallas, TX, USA, 4-9 August 2013.

20. Lim, C.P.; Woo, S.C. Text-dependent speaker recognition using wavelets and neural networks. Soft Comput. 2007, 11, 549-556. [CrossRef]

21. Park, T.J.; Kanda, N.; Dimitriadis, D.; Han, K.J.; Watanabe, S.; Narayanan, S. A review of speaker diarization: Recent advances with deep learning. Comput. Speech Lang. 2022, 72, 101317. [CrossRef]

22. Shi, Y.; Zhang, W.-Q.; Cai, M.; Liu, J. Efficient One-Pass Decoding with NNLM for Speech Recognition. IEEE Signal Process. Lett. 2014, 21, 377-381. [CrossRef]

23. Naeini, M.P.; Moshiri, B.; Araabi, B.N.; Sadeghi, M. Learning by abstraction: Hierarchical classification model using evidential theoretic approach and Bayesian ensemble model. Neurocomputing 2014, 130, 73-82. [CrossRef]

24. Dhanalakshmi, P.; Palanivel, S.; Ramalingam, V. Classification of audio signals using SVM and RBFNN. Expert Syst. Appl. 2009, 36, 6069-6075. [CrossRef]

25. Sarimveis, H.; Doganis, P.; Alexandridis, A. A classification technique based on radial basis function neural networks. Adv. Eng. Softw. 2006, 37, 218-221. [CrossRef]

26. Thasleema, T.M.; Prajith, P.; Narayanan, N.K. Time-domain non-linear feature parameter for consonant classification. Int. J. Speech Technol. 2012, 15, 227-239. [CrossRef]

27. Reddy, V.R.; Rao, K.S. Two-stage intonation modeling using feedforward neural networks for syllable based text-to-speech synthesis. Comput. Speech Lang. 2013, 27, 1105-1126. [CrossRef]

28. Kumar, R.K.S.; Lajish, V.L. Phoneme recognition using zerocrossing interval distribution of speech patterns and ANN. Int. J. Speech Technol. 2013, 16, 125-131. [CrossRef]

29. Jaitly, N.; Nguyen, P.; Senior, A.; Vanhoucke, V. Application of pretrained deep neural networks to large vocabulary speech recognition. In Proceedings of the 13th Annual Conference of the International Speech Communication Association 2012 (INTERSPEECH 2012), Portland, OR, USA, 9-13 September 2012.

30. Narendra, N.P.; Rao, K.S. Parameterization of Excitation Signal for Improving the Quality of HMM-Based Speech Synthesis System. Circuits Syst. Signal Process. 2017, 36, 3650-3673. [CrossRef]

31. Świetlicka, I.; Kuniszyk-Jóźkowiak, W.; Smołka, E. Hierarchical ANN system for stuttering identification. Comput. Speech Lang. 2013, 27, 228-242. [CrossRef]

32. Szczurowska, I.; Kuniszyk-Jóźkowiak, W.; Smołka, E. Speech nonfluency detection using Kohonen networks. Neural Comput. Appl. 2009, 18, 677-687. [CrossRef]

33. Ritchings, R.; McGillion, M.; Moore, C. Pathological voice quality assessment using artificial neural networks. Med. Eng. Phys. 2002, 24, 561-564. [CrossRef]

34. Godino-Llorente, J.; Fraile, R.; Sáenz-Lechón, N.; Osma-Ruiz, V.; Gómez-Vilda, P. Automatic detection of voice impairments from text-dependent running speech. Biomed. Signal Process. Control 2009, 4, 176-182. [CrossRef]

35. Khara, S.; Singh, S.; Vir, D. A comparative study of the techniques for feature extraction and classification in stuttering. In Proceedings of the 2018 Second International Conference on Inventive Communication and Computational Technologies (ICICCT), Ranganathan Engineering College, Coimbatore, India, 20-21 April 2018.

36. Kourkounakis, T.; Hajavi, A.; Etemad, A. FluentNet: End-to-End Detection of Stuttered Speech Disfluencies with Deep Learning. IEEE/ACM Trans. Audio Speech Lang. Process. 2021, 29, 2986-2999. [CrossRef]

37. Gupta, D.; Bansal, P.; Choudhary, K. The state of the art of feature extraction techniques in speech recognition. In Proceedings of the 50th Annual Convention of Computer Society of India, New Delhi, India, 2-5 December 2015.

38. Arbajian, P.; Hajja, A.; Raś, Z.W.; Wieczorkowska, A.A. Effect of speech segment samples selection in stutter block detection and remediation. J. Intell. Inf. Syst. 2019, 53, 241-264. [CrossRef]

39. Mahesha, P.; Vinod, D.S. Gaussian mixture model based classification of stuttering dysfluencies. J. Intell. Syst. 2015, 25, 387-399. [CrossRef] 
40. Esmaili, I.; Dabanloo, N.J.; Vali, M. Automatic classification of speech dysfluencies in continuous speech based on similarity measures and morphological image processing tools. Biomed. Signal Process. Control 2016, 23, 104-114. [CrossRef]

41. Narendra, N.; Alku, P. Dysarthric speech classification from coded telephone speech using glottal features. Speech Commun. 2019, 110, 47-55. [CrossRef]

42. Momo, N.; Abdullah; Uddin, J. Speech recognition using feed forward neural network and principle component analysis. In Proceedings of the 4th International Symposium on Signal Processing and Intelligent Recognition Systems, Bangalore, India, 19-22 September 2018; Springer: Berlin, Germany, 2019.

43. Raitio, T.; Suni, A.; Vainio, M.; Alku, P. Comparing glottal-flow-excited statistical parametric speech synthesis methods. In Proceedings of the 2013 IEEE International Conference on Acoustics, Speech and Signal Processing, Vancouver, BC, Canada, 26-31 May 2013.

44. Abolhassani, A.H.; Selouani, S.-A.; O'Shaughnessy, D. Speech enhancement using PCA and variance of the reconstruction error in distributed speech recognition. In Proceedings of the 2007 IEEE Workshop on Automatic Speech Recognition \& Understanding, Kyoto, Japan, 9-13 December 2007.

45. Chien, J.-T.; Ting, C.-W. Speaker identification using probabilistic PCA model selection. In Proceedings of the 8th International Conference on Spoken Language Processing INTERSPEECH 2004, Jeju Island, Korea, 4-8 October 2004.

46. Jolliffe, I.T. Principal Component Analysis, 2nd ed.; Springer Series in Statistics; Springer: Berlin, Germany, 2002.

47. Jhawar, G.; Nagraj, P.; Mahalakshmi, P. Speech disorder recognition using MFCC. In Proceedings of the 2016 International Conference on Communication and Signal Processing (ICCSP), Melmaruvathur, India, 6-8 April 2016.

48. Gupta, S.; Shukla, R.S.; Shukla, R.K.; Verma, R. Deep Learning Bidirectional LSTM based Detection of Prolongation and Repetition in Stuttered Speech using Weighted MFCC. Int. J. Adv. Comput. Sci. Appl. 2020, 11, 1-12. [CrossRef]

49. Bishop, C.M. Neural Networks for Pattern Recognition; Oxford University Press: Oxford, UK, 1995.

50. Tarkowski, Z. Stuttering; Kobosko, J., Ed.; PWN: Warsaw, Poland, 1999. (In Polish)

51. Cordes, A.K. Individual and Consensus Judgments of Disfluency Types in the Speech of Persons Who Stutter. J. Speech Lang. Hear. Res. 2000, 43, 951-964. [CrossRef]

52. Winursito, A.; Hidayat, R.; Bejo, A. Improvement of MFCC feature extraction accuracy using PCA in Indonesian speech recognition. In Proceedings of the 2018 International Conference on Information and Communications Technology (ICOIACT), Yogyakarta, Indonesia, 6-7 March 2018.

53. Rasheed, J.; Hameed, A.A.; Ajlouni, N.; Jamil, A.; Özyavaş, A.; Orman, Z. Application of Adaptive Back-Propagation Neural Networks for Parkinson's Disease Prediction. In Proceedings of the 2020 International Conference on Data Analytics for Business and Industry: Way Towards a Sustainable Economy (ICDABI), Sakheer, Bahrain, 26-27 October 2020.

54. Rahman, M.A.; Hossain, M.F.; Hossain, M.; Ahmmed, R. Employing PCA and t-statistical approach for feature extraction and classification of emotion from multichannel EEG signal. Egypt. Inform. J. 2020, 21, 23-35. [CrossRef]

55. Pandya, S.; Ghayvat, H. Ambient acoustic event assistive framework for identification, detection, and recognition of unknown acoustic events of a residence. Adv. Eng. Inform. 2021, 47, 101238. [CrossRef]

56. Ghayvat, H.; Pandya, S.; Patel, A. Deep learning model for acoustics signal based preventive healthcare monitoring and activity of daily living. In Proceedings of the 2nd International Conference on Data, Engineering and Applications (IDEA), Bhopal, India, 28-29 February 2020.

57. Kourkounakis, T.; Hajavi, A.; Etemad, A. Detecting multiple speech disfluencies using a deep residual network with bidirectional Long Short-Term Memory. In Proceedings of the ICASSP 2020-2020 IEEE International Conference on Acoustics, Speech and Signal Processing (ICASSP), Barcelona, Spain, 4-8 May 2020.

58. Tibrewal, V.; Haque, M.M.; Pandey, A.; Manimozhi, M. Identifying stuttering using deep learning. Int. J. Innov. Technol. Explor. Eng. 2019, 8, 1152-1154. 